\title{
Microfluidic detection and analysis by integration of thermocapillary actuation with a thin-film optical waveguide
}

\author{
Joseph P. Valentino \\ Department of Electrical Engineering, Princeton University, Princeton, New Jersey 08544 \\ Sandra M. Troian ${ }^{\text {a) }}$ \\ Department of Chemical Engineering, Princeton University, Princeton, New Jersey 08544 \\ Sigurd Wagner \\ Department of Electrical Engineering, Princeton University Princeton, New Jersey 08544
}

(Received 12 January 2005; accepted 28 March 2005; published online 25 April 2005)

\begin{abstract}
We demonstrate a nonintrusive optical method for microfluidic detection and analysis based on evanescent wave sensing. The device consists of a planar thin-film waveguide integrated with a microfluidic chip for directed surface flow. Microliter droplets are electronically transported and positioned over the waveguide surface by thermocapillary actuation. The attenuated intensity of propagating modes is used to detect droplet location, to monitor dye concentration in aqueous solutions, and to measure reaction rates with increasing surface temperature for a chromogenic biochemical assay. This study illustrates a few of the capabilities possible by direct integration of optical sensing with surface-directed fluidic devices. (c) 2005 American Institute of Physics.
\end{abstract}

[DOI: $10.1063 / 1.1922075]$

Microfluidic devices offer tremendous potential for electrical, optical, and spectroscopic interrogation of nanoliter liquid samples. Devices based on directed surface flow induced by electrowetting, ${ }^{1-4}$ dielectrophoresis, ${ }^{5,6}$ or thermocapillary actuation ${ }^{7-9}$ permit reconfigurable instruction sets for liquid positioning and mixing sequences on glass or silicon substrates. What requires further development are in situ sensor arrays for autonomous manipulation of sample location, volume, temperature, speed, and composition. Direct integration of miniaturized sensors enables application of the feedback control necessary for automated use of labon-a-chip technologies.

Our laboratory has previously demonstrated two methods ${ }^{10,11}$ for droplet detection and sensing that make use of the coplanar microelectrode arrays used for thermocapillary actuation. ${ }^{7-9}$ The first method ${ }^{10}$ monitors the rise time of embedded microheaters, from which can be extracted droplet location, volume, or composition due to changes in thermal conductivity. The second method records the capacitance change induced by an overlying droplet. The rapid response for electrode widths comparable to the liquid film thickness allows detection of droplet position, volume, composition, and evaporative loss for nanoliter samples. These sensing techniques, which probe samples by thermal or electric fields, are not suitable for all applications. Integrated optical and spectroscopic probes, such as evanescent wave sensing (EWS), offer a number of advantages in this respect. Previous studies of EWS have used external pumps to generate a continuous stream of liquid through flow cells ${ }^{12-15}$ that contact and cover the entire beam path. However, hermetically sealed channels ${ }^{16,17}$ placed against a waveguide surface produce large coupling losses and small signal-to-noise ratios. Interferometric analysis and measurement of coupling angles also increases device complexity. ${ }^{18,19}$ In this paper we dem-

a) Also at: California Institute of Technology, Pasadena, CA; electronic mail:
stroian@ princeton.edu onstrate detection and analysis of discrete droplets or rivulets that are in direct contact with only a fraction of the waveguide surface. The thin-film waveguide is directly integrated with a planar fluidic device based on thermocapillary actuation. This system registers droplet position, sample concentration, and thermal dependence of kinetic rate constants using a miniaturized low power platform. ${ }^{8,9}$ The design also offers high sensitivity with few additional fabrication steps and is especially well suited to any fluidic device based on droplet manipulation by capillary forces.

Conventional thin-film waveguides ${ }^{20-23}$ are based on light propagation by total internal reflection in an optically dense film of refractive index $n_{2}$ and thickness $d$ situated in between a supporting substrate $\left(n_{3}\right)$ and a uniform superstrate $\left(n_{1}\right)$, where $n_{2}>n_{1}$ and $n_{3}$, as in Fig. 1(a). Light propagation is restricted to specific internal angles $\theta$ satisfying the eigenvalue condition ${ }^{24} K_{2} d-\tan ^{-1}\left(K_{1} / K_{2}\right)-\tan ^{-1}\left(K_{3} / K_{2}\right)$ $=m \pi$, where $m=0,1,3, \ldots$ is the mode order. For TM modes of wavelength $\lambda, K_{i}^{\mathrm{TM}}=\left(2 \pi / \lambda n_{i}^{2}\right) \sqrt{\left|\left(n_{2} \sin \theta\right)^{2}-n_{i}^{2}\right|}$. Reflections at the interfaces generate exponentially decaying evanescent waves with a penetration distance for UV and visible light less than $100 \mathrm{~nm}$. The integrated absorbance per unit distance $D$ of adsorbing medium at the waveguidesuperstrate interface is given by $A / D=\log \left(I_{\text {inc }} / I_{\text {trans }}\right)$ $=\epsilon c(N / D) L$, where $I$ is the incident or transmitted light intensity, $\epsilon$ the molar absorptivity of the liquid sample, $c$ the liquid molarity, $N$ the number of reflections at the interface as determined from ray optics, and $L$ the evanescent path length within the liquid. ${ }^{23}$ In the present work, the presence of a droplet in the optical beam path leads to a non-uniform superstrate refractive index $\left(n_{1}\right.$ and $\left.n_{\text {liq }}\right)$, which causes detectable intensity modulation by reflective losses and attenuation of higher-order modes. ${ }^{25}$

As shown in Fig. 1(a), the substrate consists of a $0.7 \mathrm{~mm}$ thick Corning 1737 glass slide patterned by e-beam evaporation with an array of titanium heaters (50 nm thickness) of length $3 \mathrm{~mm}$, width $0.8 \mathrm{~mm}$, and spacing $60 \mu \mathrm{m}$. A $2 \mu \mathrm{m}$ 
(a)

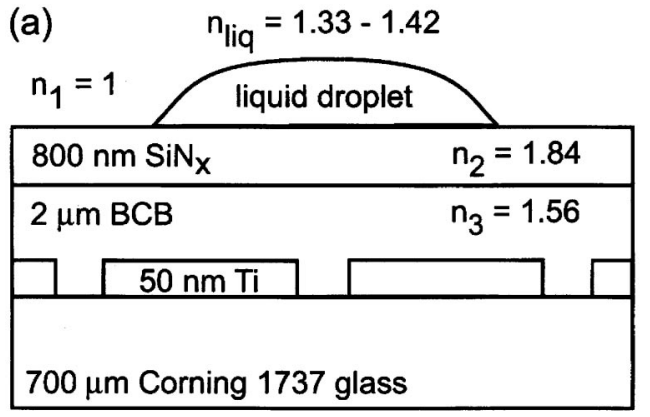

(b)

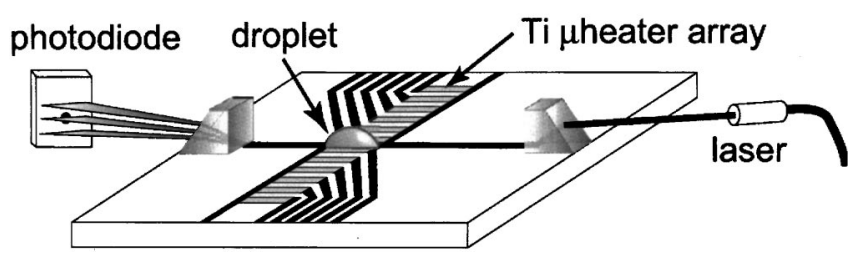

FIG. 1. (a) Cross section of device layout. (b) Plan view of microheater array (black stripes) with droplet positioned within optical beam.

thick polymer film (Cyclotene ${ }^{\mathrm{TM}} \mathrm{BCB}, n_{3}=1.561$ at $\lambda$ $=632.8 \mathrm{~nm}$ ) is spun coated onto the surface and cured at $250{ }^{\circ} \mathrm{C}$ for $2 \mathrm{~h}$. This film helps passivate and planarize the microheater surface to reduce scattering effects. The waveguide is formed by plasma-enhanced chemical vapor deposition of an $800 \mathrm{~nm}$ layer of $\mathrm{SiN}_{x}$ and the surface patterned with a monolayer of perfluoro-octyltrichlorosilane to create $2 \mathrm{~mm}$ wide wettable stripes oriented normal to the beam path. The wettable stripes laterally confine the liquid during motion. A pair of small coupling prisms (SFL57 glass, $n_{\text {prism }}=1.84$ ) are secured to the $\mathrm{SiN}_{x}$ surface by Delrin ${ }^{\circledR}$ tipped spring plungers and the substrate attached to an X-Y microscope translation stage. The waveguide refractive index, number of propagating modes (3) and coupling angles were determined via the prism coupling method. ${ }^{26}$ TMpolarized light from a He-Ne laser $(\lambda=632.8 \mathrm{~nm}, 5 \mathrm{~mW})$ is coupled into a single mode polarization-preserving optical fiber, whose orientation is controlled by a goniometer to ensure coupling to the $m=1$ mode. Scattering and reflective losses caused some propagation of the $m=0$ and 2 modes. The transmitted intensity is measured by a photodetector placed flush against the outgoing prism surface and a lock-in amplifier displays the output voltage.

Liquid droplets are transported across the beam path by thermocapillary actuation. ${ }^{7}$ An array of embedded addressable thin-film heaters generates the requisite surface temperature $T(x),{ }^{8,9}$ which in turn regulates the liquid surface tension $\gamma(x)$. The thermocapillary stress $\tau(x)$ is therefore modulated electronically to position and transport droplets (toward cooler regions) with an approximate speed, $v$ $\sim h(x) \tau / \mu$, where $h(x)$ is the droplet thickness profile in the direction of flow, $\mu(T)$ is the viscosity and $\tau=(d \gamma / d T)$ $\times(d T / d x)$ is the surface shear stress. Similar modulation is possible in two dimensions. In this study, the aspect ratio for droplet height to length was approximately 0.05 and $\nabla T$ $\approx 7^{\circ} \mathrm{C} / \mathrm{min}$. Figure 1 (b) illustrates the positioning of a droplet over the beam path by sequential activation of the microheater electrodes.

Figure 2(a) shows the voltage output for a $500 \mathrm{nl}$ droplet of dodecane and water crossing the beam path. The droplets Downloaded 15 Sep 2006 to 131.215.240.9. Redistribution subject
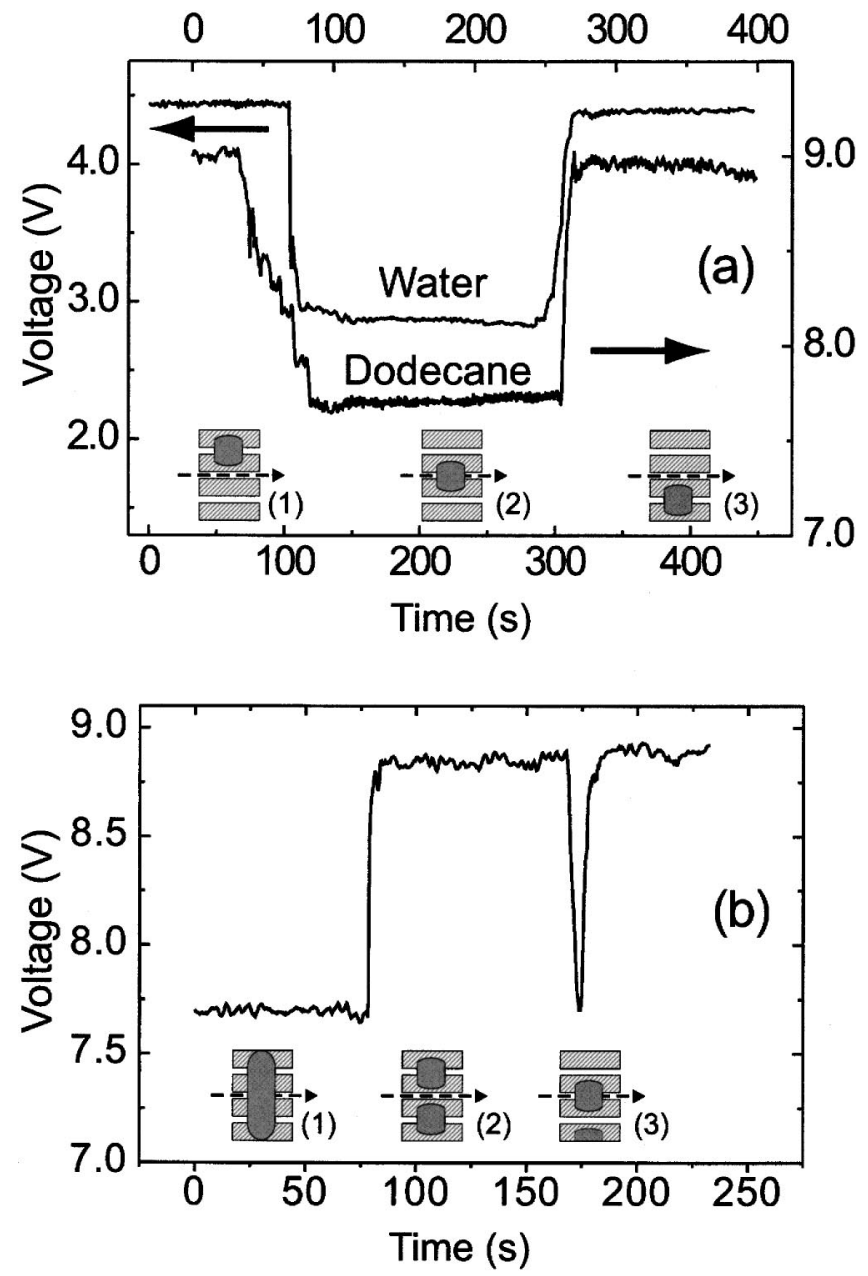

FIG. 2. (a) Voltage response during traversal of $500 \mathrm{nl}$ droplet over the optical beam path. (b) Voltage response for liquid splitting and advance of individual segments away from optical beam. Sequence in bottom panels shows corresponding droplet position controlled by activation of embedded electrodes (gray stripes). Dashed arrow designates direction of the optical beam.

were programmed to (1) approach at a speed $250 \mu \mathrm{m} / \mathrm{s},(2)$ remain in position for $3 \mathrm{~min}$, and (3) advance away at a speed $250 \mu \mathrm{m} / \mathrm{s}$. Calculations based on a configuration as in Fig. 2(a) using BeamProp $\AA^{27}$ indicate that scattering effects caused by the droplet edges are primarily responsible for signal attenuation. Figure 2(b) represents the voltage output for the scission of an elongated droplet into two identical volumes. The elongated sample was first positioned over the beam path. Heater activation beneath the midsection caused thinning and splitting by thermocapillary forces. The two segments were then moved away from the beam path at a constant speed. These sequences are sketched in the bottom panels of Fig. 2(a) and (b).

Figure 3 illustrates the voltage response for $400 \mathrm{nl}$ water droplets of different concentrations of FD \& C Blue 1 dye $\left(\epsilon=1.38 \times 10^{5} \mathrm{M}^{-1} \mathrm{~cm}^{-1}\right)$. Here $V$ and $V_{o}$ represent the output voltage for droplets with and without adsorbing dye, respectively. The excellent correspondence with the LambertBeer law, for which the absorbance is linearly proportional to $\epsilon \times c$, yields an estimate of $L=20 \mu \mathrm{m}$ (TM1 mode). ${ }^{22}$ Recovery of the baseline value after droplet traversal confirms that no dye absorbed to the waveguide surface. The limit of detection for this prototype was $3.8 \mu \mathrm{M}$, as computed from three times the root mean square of signal noise.

o AIP license or copyright, see http://apl.aip.org/apl/copyright.jsp 


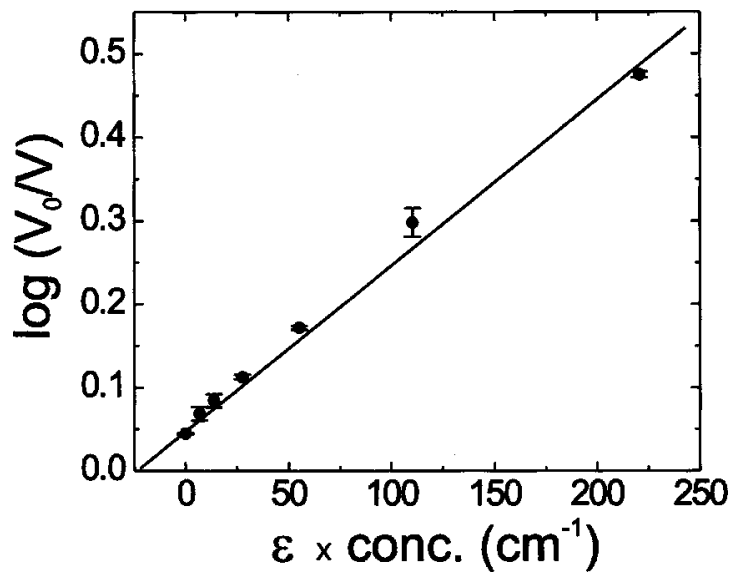

FIG. 3. Normalized voltage response showing evanescent wave absorption by $400 \mathrm{nl}$ water droplet containing different concentrations of FD \& C Blue 1 dye. Data points represent an average of three independent measurements.

The device was also used to measure the increase in reaction rate with temperature for the hydrolysis of 5-bromo4-chloro-3-indolyl- $\beta$-D-galactoside $(X$-gal) by the enzyme $\beta$-galactosidase $(\beta$-gal) into galactose and an insoluble indigo precipitate ${ }^{28,29}$ This assay is commonly used in analyzing cell lineages and gene expression patterns in developing tissues of eukaryotes. One $400 \mathrm{nl}$ droplet of a phosphate buffer containing $\beta$-gal was made to coalesce with a second $400 \mathrm{nl}$ droplet of $200 \mathrm{mM} \mathrm{X}$ - gal in dimethylsulfoxide at substrate surface temperatures $T_{\text {surface }}=22$ and $29^{\circ} \mathrm{C}$. Figure 4 represents the attenuated signal as a function of time. Initially, the $\beta$-gal primarily converts $X$-gal to the indigo precipitate. As more precipitate forms, the reverse reaction proceeds until equilibrium is attained. The increase in surface temperature leads to a doubling of the reaction rate. The small variations in output intensity at early times can be traced to fluctuations during droplet positioning prior to reaction.

In summary, these results represent a few of the capabilities made possible by integration of evanescent wave

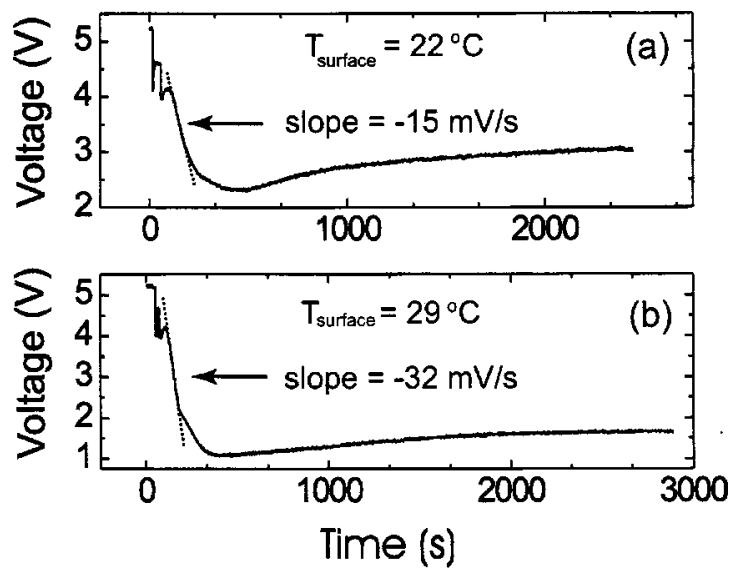

FIG. 4. (a) Voltage response from production of indigo precipitate formed during enzymatic hydrolysis of $\mathrm{X}$-gal at substrate surface temperatures (a) $T_{\text {surface }}=22^{\circ} \mathrm{C}$ and (b) $T_{\text {surface }}=29^{\circ} \mathrm{C}$. sensing with thermocapillary actuation. This device can be further miniaturized by substitution of diffraction grating couplers and the sensitivity improved by investigating the attenuation of each separate mode. These refinements are underway along with a theoretical analysis to assess the effect of droplet shape and nonuniform superstrate refractive index on mode propagation and scattering.

This work was funded by the NASA Microgravity Fluid Physics Program and the US Army TACOM-ARDEC. One of the authors (J. P. V.) thanks Dr. P. K. Tien of Bell Labs for helpful discussions. SMT gratefully acknowledges the hospitality and financial support of the Moore Distinguished Scholar Program at the California Institute of Technolgy.

${ }^{1}$ J. Lee and C. J. Kim, J. Microelectromech. Syst. 9, 171 (2000).

${ }^{2}$ M. G. Pollack, R. B. Fair, and A. D. Shenderov, Appl. Phys. Lett. 77, 1725 (2000).

${ }^{3}$ M. G. Pollack, A. D. Shenderov, and R. B. Fair, Lab Chip 2, 965 (2002).

${ }^{4}$ S. K. Cho, H. J. Moon, and C. J. Kim, J. Microelectromech. Syst. 12, 70 (2003).

${ }^{5}$ T. B. Jones, M. Gunji, M. Washizu, and M. J. Feldman, J. Appl. Phys. 89, 1441 (2001).

${ }^{6}$ R. Ahmed, D. Hsu, C. Bailey, and T. B. Jones, Microscale Thermophys. Eng. 8, 271 (2004).

${ }^{7}$ A. A. Darhuber, J. M. Davis, S. M. Troian, and W. W. Reisner, Phys. Fluids 15, 1295 (2003).

${ }^{8}$ A. A. Darhuber, J. P. Valentino, J. M. Davis, S. M. Troian, and S. Wagner, Appl. Phys. Lett. 82, 657 (2003).

${ }^{9}$ A. A. Darhuber, J. P. Valentino, S. M. Troian, and S. Wagner, J. Microelectromech. Syst. 12, 873 (2003).

${ }^{10}$ A. A. Darhuber, S. M. Troian, and S. Wagner, J. Appl. Phys. 91, 5686 (2002).

${ }^{11}$ J. Z. Chen, A. A. Darhuber, S. M. Troian, and S. Wagner, Lab Chip 4, 3 (2004).

${ }^{12}$ T. E. Plowman, J. D. Durstchi, H. K. Wang, D. A. Christensen, J. N. Herron, and W. M. Reichert, Anal. Chem. 71, 4344 (1999).

${ }^{13}$ E. Verpoorte, Lab Chip 3, 42N (2003).

${ }^{14}$ K. Misiakos, S. E. Kakabakos, P. S. Petrou, and H. H. Ruf, Anal. Chem. 76, 1366 (2004).

${ }^{15}$ R. Bernini, S. Campopiano, L. Zeni, and P. M. Sarro, Sens. Actuators B 100, 143 (2004).

${ }^{16}$ K. B. Mogensen, J. El-Ali, A. Wolff, and J. P. Kutter, Appl. Opt. 42, 4072 (2003).

${ }^{17}$ Z. Wang, J. El-Ali, M. Engelund, T. Gotsaed, I. R. Perch-Nielsen, K. B. Mogensen, D. Snakenborg, J. P. Kutter, and A. Wolff, Lab Chip 4, 372 (2004).

${ }^{18}$ Ch. Stamm and W. Lukosz, Sens. Actuators B 11, 177 (1993).

${ }^{19}$ S. C. Jakeway and A. J. de Mello, Analyst (Cambridge, U.K.) 126, 1505 (2001).

${ }^{20}$ J. E. Midwinter, IEEE J. Quantum Electron. QE-7, 339 (1971).

${ }^{21}$ G. L. Mitchell, IEEE J. Quantum Electron. QE-13, 173 (1977).

${ }^{22}$ M. D. DeGrandpre, L. W. Burgess, P. L. White, and D. S. Goldman, Anal. Chem. 62, 2012 (1990).

${ }^{23}$ S. S. Saavedra and W. M. Reichert, Anal. Chem. 62, 2251 (1990).

${ }^{24}$ D. Marcuse, Theory of Dielectric Optical Waveguides (Academic, San Diego, 1991).

${ }^{25}$ S. S. Saavedra and W. M. Reichert, Appl. Spectrosc. 44, 1210 (1990).

${ }^{26}$ R. Ulrich and R. Torge, Appl. Opt. 12, 2901 (1973).

${ }^{27}$ BeamProp V5.1.5 (RSoft Design Group, Inc., Ossining, NY, 2004).

${ }^{28}$ J. Sambrook, E. F. Fritsch, and T. Maniatis, Molecular Cloning: A Laboratory Manual, 2nd ed. (Cold Spring Harbor, NY, 1989).

${ }^{29}$ G. R. MacGregor, G. P. Nolan, S. Fiering, M. Roederer, and L. A. Herzenberg, in Methods in Molecular Biology, edited by E. J. Murray (Humana, Clifton, NJ, 1991), Vol. 7, Chap. 17. 